\title{
Seven integrated influenza surveillance systems in Taiwan
}

\author{
Chwan-Chuen King ${ }^{\mathrm{a}, \mathrm{b}, *}$, Chuan-Liang Kao ${ }^{\mathrm{c}}$, Ding-Ping Liu ${ }^{\mathrm{d}}$, \\ Min-Chu Cheng ${ }^{\mathrm{e}}$, Hui-Lin Yen ${ }^{\mathrm{a}, \mathrm{b}}$, Min-Shiuh Lee, \\ Ching-Ping Tsai ${ }^{\text {, }}$, Shin-Ru Shih ${ }^{\mathrm{h}}$, Happy K. Shieh ${ }^{\mathrm{f}}$, \\ Jen-Pang Hsiu ${ }^{d}$, Shu-Fang Li ${ }^{d}$, Hour-Young Chen ${ }^{d}$, Hsu-Mei Hsu ${ }^{d}$, \\ Shing-Jer Twu ${ }^{\mathrm{d}}$, Nancy J. Cox ${ }^{\mathrm{i}}$, Robert G. Webster ${ }^{\mathrm{j}, \mathrm{k}}$ \\ a Institute of Epidemiology, College of Public Health, National Taiwan University (NTU), Taipei, Taiwan \\ ${ }^{\mathrm{b}}$ Takemi Program in International Health, School of Public Health, Harvard University, Boston, MA 02115, USA \\ ${ }^{\mathrm{c}}$ School of Medical Technology, College of Medicine, NTU and Division of Virology, \\ Department of Laboratory Medicine, NTU Hospital, Taipei, Taiwan \\ ${ }^{\mathrm{d}}$ Centers for Disease Control, Taipei, Taiwan \\ ${ }^{\mathrm{e}}$ Council of Agriculture, Animal Health Research Institute, Taipei, Taiwan \\ ${ }^{\mathrm{f}}$ National Chung Hsing University, Taichung, Taiwan \\ ${ }^{\mathrm{g}}$ Pig Research Institute in Taiwan, Taiwan \\ ${ }^{\mathrm{h}}$ Department of Medical Technology, Chang-Gung University, Taoyuan, Taiwan \\ influenza Branch, Centers for Disease Control and Prevention, Atlanta, USA \\ ${ }^{\mathrm{j}}$ Department of Virology and Molecular Biology, St. Jude Children's Research Hospital, Memphis, TN, USA \\ ${ }^{\mathrm{k}}$ WHO Collaborating Center for Studies on the Ecology of Influenza in Animals and Birds, USA
}

\begin{abstract}
Background: Influenza surveillance in recent decades in Taiwan showed that the major virus isolates were A/Hong Kong/68-like (H3N2), A/England/42/72-like (H3N2), A/Taiwan/1/86 (H1N1), A/Texas/36/91-like (H1N1), A/Taiwan/112/96 (H1N1), and A/Taiwan/118/96 (H1N1) based on passive virologic surveillance before 1999. Materials and methods: To prevent the potential public health threat of a newly emerged influenza A strain like H5N1 in Hong Kong, seven surveillance systems have been established in Taiwan since 1999, including severe case reporting, sentinel physician, contract-laboratory, poultry market, domestic avian, wild bird and pig surveillance. Scientific exchanges and possible public health actions are discussed in regular meetings during the winter season. Results: The isolation rates of influenza A virus were higher among ducks than
\end{abstract}

\footnotetext{
* Corresponding author. Institute of Epidemiology, College of Public Health, National Taiwan University, 1 Jen-Ai Road Section 1, Taipei, Taiwan. Tel.: +886-2-2341-4347; fax: +886-2-2351-1955.

E-mail address: a1234567@ccms.ntu.edu.tw (C.C. King).
} 
chickens in both domestic avian and market surveillance systems. Ten subtypes of influenza A virus can be found in migrating wild birds, which may serve as a reservoir for the genetic and antigenic changes of the virus. The H6 subtype was isolated from both wild birds and market surveillance systems. Pig surveillance found that $\mathrm{H} 3$ occurred more in the winter, whereas $\mathrm{H} 1$ appeared predominantly in the summer. Human virologic surveillance also demonstrated summer and winter peaks. Market surveillance in Taipei showed H6, H4, and H3 subtypes isolated from ducks only. Sentinel physician surveillance experience has taught us the importance of involving local clinics, particularly in farm areas. The traditional passive physician reporting surveillance provided limited information and it was replaced by severe case reporting. Most elderly physicians in Taiwan did not like to report influenza even after implementation of direct electronic reporting through a worldwide web system. More modern technology will be applied to increase the timeliness and representativeness of influenza surveillance in preparing for future pandemics. Conclusion: Integration of animal and human influenza surveillance systems in the high population density in Taiwan serves as a model in the efforts of global influenza surveillance to detect newly emerged influenza virus strains via several channels, particularly useful in detecting severity of cases and inter-species transmission. (C) 2001 Elsevier Science B.V. All rights reserved.

Keywords: Surveillance; Influenza A; Taiwan; Interspecies transmission

\section{Introduction}

Pandemic influenza is caused by new human influenza A virus which arises due to genetic reassortment of animal influenza viruses or direct intra-species transmission and has global public health significance [1]. In addition, epidemic influenza is responsible for respiratory morbidity, hospitalization and mortality worldwide [2-4]. The impact of the next influenza A pandemic on the USA has been estimated as 89,000-207,000 deaths, 314,000-734,000 hospitalized cases, 18-42 million clinical visits, 20-47 million additional people ill and 71-166 billion American dollars in social disruption [5]. Surveillance is the most effective public health prevention and control strategy by providing early detection of an epidemic, defining high risk populations in a given year, investigating the extent and distribution of severe cases, identifying the current circulating subtypes of the virus, monitoring for antigenic shift, tracing the origin of the newly emergent/re-emergent virus, and developing vaccines for immunization programs [6-9].

Surveillance involves the systematic collection, consolidation, and evaluation of morbidity and mortality reports and other relevant data such as risk or preventive factors [10]. Public health surveillance has a responsibility for disease control activities, which is distinct from epidemiologic surveillance, that focuses mainly on epidemiologic research. In general, public health professionals at local, state/provincial, national and international levels regularly analyze surveillance data and use this information for taking appropriate action [11-14]. Effective disease control and prevention programs depend primarily on sensitive and representative surveillance systems to detect infectious disease problems early enough to take an immediate action [15]. In particular, influenza surveillance requires close collaboration both at the various levels of the health system within a country and between countries in order to take immediate global efforts to prevent pandemic. Since it was established in 1947, the WHO global 
surveillance for influenza has grown to 110 designated National Influenza Centers in 80 countries [16]. There are many different national influenza surveillance systems, however, most of these are directed primarily at the detection of human cases and few of them are integrated with animal influenza surveillance systems in order to fully understand the ecology, evolution and mechanisms contributing to the emergence of virulent influenza A viruses.

Taiwan is located in a subtropical area of Southeast Asia with an extremely high population density in most urban areas, particularly the capital City of Taipei. Therefore, respiratory infections including influenza have a public health priority. The incentive to improve infectious disease surveillance systems in this area has attracted more attention since a large-scale epidemic of enterovirus 71 occurred in Taiwan in 1998. In addition, frequent travel by Taiwanese to Hong Kong, mainland China and other Southeast Asian countries, where the origin of several pandemics of flu had been documented in the past, have also provided an impetus to monitor influenza A virus activity by establishing sensitive, representative and integrated surveillance systems. Furthermore, the epidemic of influenza A (H5N1) in Hong Kong in 1997 [17] and the finding of human H9N2 isolates in China and Hong Kong in 1999 [18,19] imply that influenza A viruses are widespread in Asian poultry. Under these circumstances of potential public health threats, we became the first country to integrate all seven influenza surveillance systems, including both human and animal surveillance, prior to the epidemic season 1999-2000. This report describes the strengths and weaknesses of each influenza surveillance system with more emphasis on our valuable experience with respect to successes, failures and future efforts.

\section{Materials and methods}

\subsection{Influenza surveillance systems in Taiwan before 1998}

Three major influenza surveillance systems had been operating from 1970s until 1998, including human virologic surveillance at the national level, domestic avian surveillance in central Taiwan and swine flu surveillance in the Institute of Pig Research in Miaoli. Unfortunately, similar to most other countries, there was little exchange of influenza A virus of activity and data analysis between animal and human influenza surveillance systems on a regular basis. In addition, prior to 1998 while influenza-reporting data were passively collected on a weekly and monthly basis, summary data was compiled annually and published in Chinese Epidemiology Bulletin. However, most Chinese physicians did not like to report influenza and only two to three sentinels located in Taipei sent regular respiratory specimens each month for virologic identification [20]. On the other hand, local Health Centers and Bureaus obtained infectious disease morbidity and mortality data passively and three different organizations (Bureau of Communicable Disease, National Institute of Preventive Medicine and National Quarantine Service) were responsible for the final infectious disease surveillance report before the large-scale epidemic of enterovirus 71 throughout Taiwan in the summer of 1998. Following this epidemic the Center for Disease Control was initiated to combine all of the above three organizations in order to increase the efficiency of infectious disease surveillance and health policy. Therefore, 
several improved surveillance systems, including severe case reporting, sentinel physician reporting and virologic laboratory reporting systems for human influenza were evaluated since then.

In animal influenza A surveillance, both pig and avian influenza surveillance systems were mainly seroepidemiologically oriented although strains of influenza A were sometimes isolated [21,22]. The pig surveillance collected nasal swabs from collaborating pig farms, whereas the avian flu surveillance, a passive system, regularly diagnosed infections such as Newcastle disease, etc., among sick chickens and ducks. In addition, influenza A viruses were occasionally isolated from organs of wild birds. However, there was no selective monitoring of migrating birds.

\subsection{Systematic changes in influenza surveillance process}

Because the elderly are at greater risk for influenza-related complications and the numbers of elderly persons have been increasing rapidly in recent years, systematic approaches to improve current human flu surveillance have become necessary in Taiwan. The transmission of $\mathrm{H} 5 \mathrm{~N} 1$ influenza viruses to humans, which resulted in six fatal cases of 18 infected persons, highlighted the role of chickens as a potential intermediate host for human infection [17,19]. Furthermore, the isolation of H9N1 influenza viruses from humans in mainland China and Hong Kong again illustrates that the most effective surveillance systems should involve animal reservoirs or intermediate hosts together with human surveillance systems [27,28]. Taiwan has more than 100,000 businessmen and travelers who visit Hong Kong and China each year. There has been little information regarding the gene pools of influenza A viruses among migrating birds and domestic avian species in Asia, therefore, seven different surveillance systems of influenza A have been integrated since 1998-1999.

\subsubsection{Wild bird virologic surveillance}

Feces of wild birds including residential and migratory birds were collected monthly from several bird roosting areas distributed all over Taiwan main island (the south, central, east, west, and north) and Kinmen isolated islet, during seasonal months when birds (wild water fowls) were flying into Taiwan. Systematic stratified sampling of 30 ducks per group and 1-2 groups were collected at each surveillance site [23]. In addition, cloacal swabs of banded birds were also obtained. These samples were inoculated directly into 10-day-old embryonated chicken eggs for the isolation of influenza virus. Subtyping of hemaglutinin ( $\mathrm{H}$ antigen) was undertaken using reference serum samples obtained from Dr. Hiroshi Kida, Hokkaido University, Japan and Dr. D.J. Alexander in NCVL, England. Sequence analysis of $\mathrm{H} 5$ and $\mathrm{H} 7$ subtypes were undertaken to determine the presence of basic amino acid sequences, which contribute to virulence of the virus.

\subsubsection{Domestic avian influenza surveillance}

Sick domestic birds were sent to Dr. Happy Shieh's laboratory at the Department of Veterinary Medicine at the National Chung-Hsing University, Taichung, Taiwan. Viruses were isolated, identified and sequenced using the primer sets designed based on the local Taiwan avian sequence data bank [24]. 


\subsubsection{Market avian virologic surveillance}

With the consultation and technical guidance from Dr. R. Webster, we initiated a market avian virologic surveillance system at the College of Public Health, National Taiwan University and selected a large wholesale market located in Taipei selling live chickens, ducks, and geese from different parts of Taiwan. Faeces of white chickens, brown chickens, black-bone chickens, and ducks were collected early in the morning once each week from November 1999 to June 2000 [25].

\subsubsection{Swine influenza surveillance}

Both serologic and virologic surveillance systems were employed to monitor the virus activity at the Pig Research Institute in MiaoLi, Taiwan. Tracheal samples were collected from pigs manifesting respiratory symptoms/signs. The six sentinel sites included two pig farms with about 4000-5000 pigs each in Northern (TaoYuan, MiaoLi), Central (Taichung, Changhwa) and Southern Taiwan (Kaohsiung, Pingtung). Specimens were inoculated into 10-day-old specific pathogen free embryonated chicken eggs. Human and pig flu A subtypes were compared frequently [26].

\subsubsection{Sentinel physician surveillance}

Since 1990 sentinel physician surveillance in Taiwan has been in place established to monitor chickenpox, measles, mumps, rubella and neonatal tetanus. Upper respiratory disease sentinel surveillance system was initiated in the 39th week of 1995 and sentinel physicians were selected according to their past reporting records and enthusiasm. The influenza-like illness (ILI) sentinel surveillance was initiated in January 1999 to predict trends and provide early warning of epidemics. The case definition of ILI includes patients with fever (ear temperature over $38{ }^{\circ} \mathrm{C}$ ), respiratory symptoms/signs and muscle pain/headache/exhaustion. Patients with rhinitis, tonsillitis and bronchiolits were excluded [27].

\subsubsection{Human virologic surveillance}

Nationwide human influenza virologic surveillance linking the sentinel physician and laboratory surveillance systems was initiated in March 1999. Specimens were inoculated in MDCK cells, immunofluorescence and hemaglutination inhibition tests were used to identify subtypes and selected strains were sent to WHO collaborating laboratories or Centers for Disease Control and Prevention in Atlanta, USA, for antigenic studies and confirmation. This system involves virology laboratories distributed in southern (Kaohsiung Medical College in Kaohsiung, ChengKung University in Tainan), central (ChangHwa Christian Hospital in Changhwa) and eastern (TzeChi Hospital in HwaLien) and northern (National Taiwan University Hospital and ChangGun Memorial Hospital in Taipei) Taiwan in July 1999 [28].

\subsubsection{Severe case surveillance}

Since many infections may have similar symptoms/signs to influenza, most physicians in Taiwan do not like to report these. The passive physician-based surveillance system had very limited values in timeliness and specificity and was replaced by a hospital based severe case surveillance in January 2000. 


\section{Results}

\subsection{Use and strengths of animal influenza surveillance systems}

Because influenza A is a zoonotic disease which may contribute new virulent human strains, information obtained from animal virologic surveillance is crucial [29].

\subsubsection{Wild bird flu surveillance}

Wild bird surveillance system provides monthly data of flu virus activity, geographic and host species distribution of different subtypes of influenza A viruses, and prevailing environmental conditions. Influenza A virus activity was strongly associated with the seasonal movement of migrating wild water ducks. Ten different $\mathrm{H}$ and $\mathrm{N}$ subtypes, including H4N2, H4N6, H3N8, H4N8, H10N7, H2N3, H7N1, H1N3, H4N7 and H1N1 were all isolated [23]. Tests indicated that none of these viruses were virulent for chickens.

One area located in northeastern Taiwan close to a duck raising farm was further investigated and monitored for their virus activity. $\mathrm{H}$ subtypes of newly isolates from domestic ducks and market surveillance have been communicated and compared frequently. Because the peaking months of bird flu virus appeared before human flu peaking months, the director of the Center for Disease Control in Taiwan in charge of human influenza prevention and control activities was immediately notified about updated bird flu surveillance data.

\subsubsection{Domestic avian flu surveillance}

The isolation rates varied in different geographic areas. Ducks in areas with potential contact of migrating birds and open farming had the higher flu A virus isolation rate. Health education and changing the farming style were then implemented by educating poultry farmers how to avoid contact with wild birds. The isolation rates of influenza A virus decreased since the widespread introduction of closed farming methods. Because sequence data currently in gene bank consists mostly of influenza A viruses isolated from European and American birds, Asian avian flu A sequences are urgently required to optimise primer design for the reverse-transcriptase polymerase chain reaction (RT-PCR) and to monitor genetic changes and their possible role in viral pathogenesis [24].

\subsubsection{Market flu surveillance}

Chinese markets selling live chickens or ducks provide the best opportunity for contact between domestic avians and the human population and potential exchange of viruses. Phylogenetic studies on all eight segments of the influenza A viruses isolated from markets provide information useful in evaluating both the possible pandemic potential of viruses, due to reassortment, and also their possible sources by sequence homology. Until to now, no influenza A virus have been isolated from chickens in Taiwan, whereas ducks had the highest influenza A virus isolation rate. Nine subtypes were isolated, including H3N1, H3N2, H3N6, H3N8, H4N1, H4N2, H4N4, H4N6 and H6N1 [25]. Sequence data for the eight gene segments were determined in Dr. Robert Webster's laboratory.

Because of public health concerns, seroepidemiologic study on the extent of infection due to these isolates was investigated among veterinarians, poultry workers and market 
salesmen. A study of 195 poultry workers and 146 veterinarians distributed throughout Taiwan were found seronegative to the H4 and H6 subtypes. Questionnaire data analysis showed that weather, altitude, number of animals raised or species were not the major influential factors of interspecies transmission.

\subsubsection{Swine flu surveillance}

Swine flu surveillance has the highest priority because Taiwan has the largest swine population in Asia. Monthly and geographic distributions of swine influenza A viruses have been monitored. For hygiene reasons contacts between humans and pigs have now been minimized by industrialization of pig farms in most areas, however, swine influenza viruses were frequently isolated. Both $\mathrm{H} 1$ and $\mathrm{H} 3$ subtypes were isolated with different subtypes predominating in different years. The monthly pattern of $\mathrm{H}$ subtypes in swine flu was consistent with those found in human influenza A in the same year in 1998-2000, even the shift from $\mathrm{H} 3$ to $\mathrm{H} 1$ subtype. There was no striking geographic variation of swine flu viruses and sequence data for $\mathrm{H}$ and $\mathrm{N}$ genes indicated the possibility of human flu $\mathrm{A}$ genes existed in pigs. Both the representativeness and specificity of this surveillance will be further improved in coming years.

\subsection{Improvement of human flu surveillance}

Several human influenza surveillance systems were compared. ILI reported by sentinel physicians and laboratory surveillance showed an increase in activity in the same month. The laboratory surveillance detected the virus activity one month earlier than previous years due to the improved sampling through the sentinels and increased numbers of sentinels.

\subsubsection{Direct computer reporting from physicians}

Due to the unwillingness of physicians to report influenza, a program for direct computer reporting was initiated in December 1999. Unfortunately, older physicians do not adapt to the computer system. Further improvement is in process.

\subsubsection{Representative of sentinel physician surveillance system}

The proportion of sentinel physicians was planned to cover $1.5 \%$ of the total Taiwan population with an aim of approximately one sentinel physician per 750 people. Sampling was based on 63 medical care network districts. The greatest number of surveillance sites was located in areas of high population density, Taipei City and County, particularly pediatricians whose patients are living or working close to market or farm areas. On the other hand, rural areas with avian or pig farms or wild birds roosting areas were considered as potential sites for the sources of newly emerging influenza viruses and surveillance sites were, therefore, recruited based not only on total population but also considering the potential sources of influenza viruses.

\subsubsection{Initiating contract laboratory virologic surveillance system}

Virology laboratories in Taiwan are mostly found in teaching hospitals. Four contract virology laboratories commenced operation in March 1999 to collect clinical specimens for isolation of influenza viruses and to extend geographic sampling to the east and central 
part of Taiwan, two more contract laboratories with fewer well-trained technical laboratory personnel were added and supervised in July 1999. A further five laboratories, including the largest military hospital in Taipei and two more hospitals in central and southern Taiwan, catering for patients from rural areas, have been invited to increase the sensitivity of virologic surveillance. Data were collected each week for rapid feedback data analysis. The highest isolation rates of influenza viruses were found in the core laboratory, which collected more clinical samples from several local clinics and had pediatricians with virologic training, than the other virologic sentinel sites. The reasons include better communication with the local physicians and trained enthusiastic pediatricians who pay much greater attention to viral diseases. Analysis indicated that most district and community hospitals where patients have more potential exposure to farm animals might not be detected by the current human virologic surveillance. More comprehensive surveillance will assist in selecting virus strains for immunization program. In 19992000 in Taiwan, there was a decrease in $\mathrm{A}(\mathrm{H} 3 \mathrm{~N} 2) \mathrm{A} / \mathrm{Sydney} / 5 / 97-$ like strains and the appearance of A/Moscow/10/99-like isolates, A(H1N1) A/New Caledonia/20/99-like strains and B/Beijing/184/93-like strains.

\subsubsection{Clinical severe case surveillance}

To avoid the possible chaos associated with the emergence of pandemic strains, a clinical severe case surveillance system was established in January 2000. Epidemiologic investigations will be initiated if a clustering of cases in place or time occurs or unusual epidemiological characteristics are seen.

\subsection{Integration of animal and human flu surveillance systems}

\subsubsection{Regular scientific exchanges}

Following consultation with Dr. Nancy Cox at the Centers of Disease Control and Prevention at Atlanta, GA, USA, regular monthly scientific exchange meetings were instituted during influenza seasonal months commencing with a meeting at the Institute of Epidemiology, National Taiwan University in December 1999. Data from each surveillance system is presented and discussed for public health actions and future research. Suggestions and future collaborative efforts were discussed with consensus.

\subsubsection{Integrated study design}

Collaborative research projects were initiated in December 1999 with more integration of study sites, selection of study populations and data analysis of different surveillance systems. In addition, both virologic isolation and seroepidemiologic investigation are complementary in determining the magnitude of infection and spread of a new subtype of influenza A virus.

\subsection{Epidemiologic trends of human flu virologic surveillance at the National Taiwan University Hospital}

The virological surveillance data at NTU Hospital showed that the predominant influenza season was winter to spring, although influenza isolates were found in late 


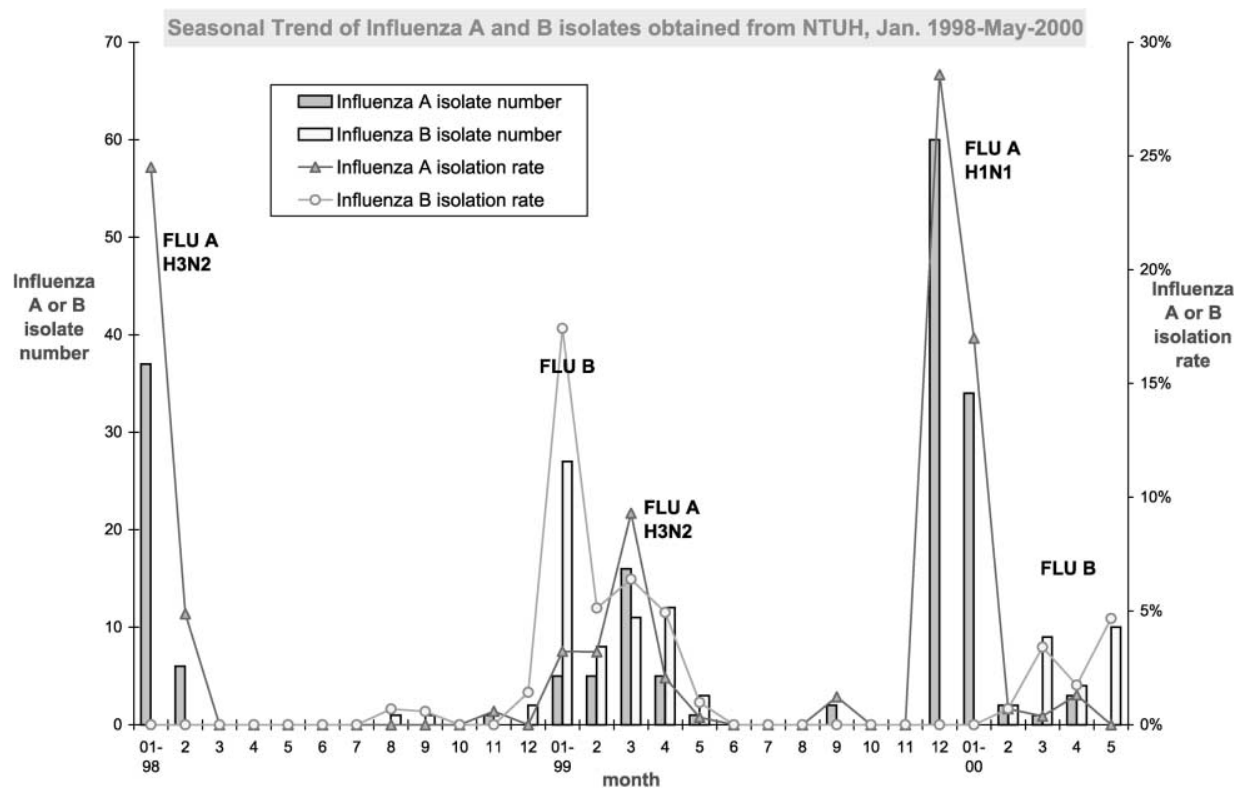

Fig. 1. Seasonal trend of influenza A and B isolates obtained from National Taiwan University Hospital, January 1998-May 2000.

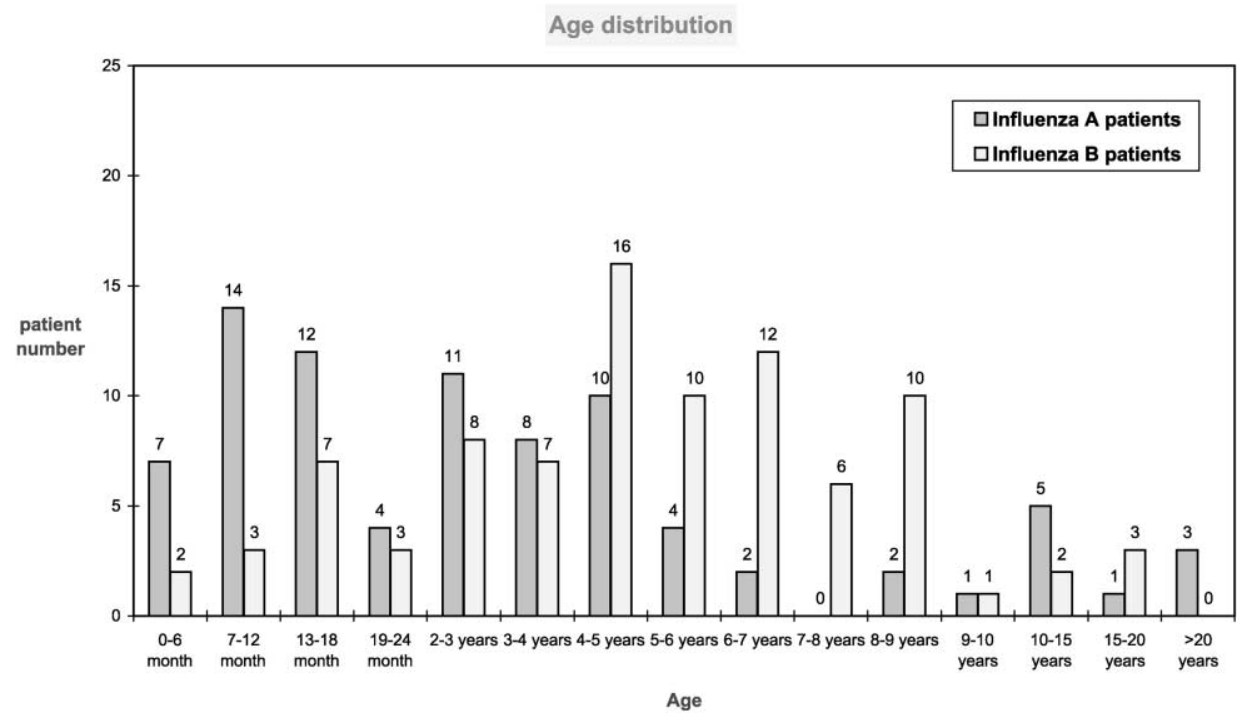

Fig. 2. Age distribution of influenza A and B patients at National Taiwan University Hospital, January $1998-$ May 2000. 
summer or autumn. Both NTU Hospital and the national virologic surveillance systems can detect influenza virus activity even during the summer time (Fig. 1). Although $\mathrm{H} 3 \mathrm{~N} 2$ and H1N1 were co-circulating in recent years in Taiwan, different serotypes of the virus were dominant in different years. Comparing the age distribution of influenza $\mathrm{A}$ and $\mathrm{B}$ patients under 10 years old, we found that influenza $\mathrm{A}$ patients were significantly younger than influenza B patients $(p<0.01)$. Age distribution of different serotypes also varies indicating that different selective pressure may exist among H3N2 versus H1N1 (Fig. 2).

\section{Discussion}

Influenza A viruses continue to evolve by antigenic drift and shift in humans, pigs and horses. Both pigs and aquatic birds may contribute to the emergence of pandemic influenza viruses for humans [29]. Therefore, a network of surveillance, which integrates both human and animal surveillance systems considering sources of new emergent influenza virus strains, intermediate hosts, inter-species transmission and mild and severe human cases, is important to public health. Such surveillance systems mean that prevention strategies for future pandemics of influenza $\mathrm{A}$ in humans become feasible [30,31]. The timeliness of reporting, representative, sensitivity, specificity, flexibility, simplicity, feed-back responses, effectiveness of coordination and the ability to detect unusual events and outbreaks in each surveillance system for etiologic agents with pandemic potential require ongoing evaluation each year.

Routine operation of surveillance must consider: (1) number and type of reporting channels and sources, (2) efficient method(s) of transmitting reporting data and relevant information, and (3) amount and type of information necessary to establish clinical and laboratory diagnosis. While active surveillance provides more timely data than passive surveillance it is time consuming. Simple surveillance systems gain the greatest compliance in data collection, efficient operation, and rapid distribution of key messages for effective prevention and control. Continuous evaluation of the process of surveillance is necessary and we have found that biweekly meetings are very helpful to facilitate changes. In addition, other surveillance systems such as emergency room surveillance, ICU surveillance, school students' absenteeism, work absenteeism and drug usage may also help to increase the sensitivity of the current surveillance systems in Taiwan.

Future efforts to improve our influenza surveillance will include: (1) application of rapid viral diagnosis tests to surveillance, particularly for rural, suburban and isolated areas where more animal-human contacts are possible [32], (2) examining the molecular evolution of $\mathrm{H} 3$ and $\mathrm{H} 1$ viruses in both humans and animals by phylogenetic analysis of all eight viral gene segments [33], (3) establishing gene pool data base in different parts of Taiwan by linking epidemiologic findings and public health policy for better decision-making, and (4) integrating our surveillance from study design, selection of sentinels, study sites and populations to data analysis. For those areas where more virulent or diverse subtypes of influenza A virus are isolated from wild waterfowl, chickens, ducks and pigs, more pediatric sentinel physicians will be added to monitor 
the subsequent virus activities in both humans, domestic avians and pigs. We believe international collaboration on flu surveillance among Asian countries and areas, particularly Hong Kong, Taiwan and China, will contribute more understanding to avoid future pandemics [31].

\section{References}

[1] R.G. Webster, Influenza: an emerging microbial pathogen, in: R.M. Krause (Ed.), Emerging Infections, Academic Press, New York, 1998, pp. 275-300.

[2] T.D. Szucs, Influenza: the roles of burden-of-illness research, PharmacoEconomics 16 (Suppl. 1) (1999) $27-32$.

[3] K.M. Sullivan, Health impact of influenza in the United States, PharmacoEconomics 9 (Suppl. 3) (1996) $26-33$.

[4] D.M. Fleming, The impact of three influenza epidemics on primary care in England and Wales, PharmacoEconomics 9 (Suppl. 3) (1996) 38-45.

[5] S. Klimov, Global influenza surveillance and vaccine strain selection, "International Conference on Emerging Infectious Diseases", July 16-19, Atlanta, Georgia, USA.

[6] Y.P. Lin, M. Shaw, V. Gregory, K. Cameron, W. Lim, A. Klimov, K. Subbarao, Y. Guan, S. Krauss, K. Shortridge, R. Webster, N. Cox, A. Hay, Avian-to-human transmission of H9N2 subtype influenza A viruses: relationship between H9N2 and H5N1 human isolates, Proc. Natl. Acad. Sci. U. S. A. 97 (17) (2000) 9654-9658.

[7] A.I. Karasin, M.M. Schutten, L.A. Cooper, C.B. Smith, K. Subbarao, G.A. Anderson, S. Carman, C.W. Olsen, Genetic characterization of H3N2 influenza viruses isolated from pigs in North America, 19771999: evidence of wholly human and reassortant virus genotypes, Virus Res. 68 (1) (2000) 71-85.

[8] Centers for Disease Control and Prevention, Delayed supply of influenza vaccine and adjunct ACIP influenza vaccine recommendations for the 2000-2001 influenza season, Advisory Committee on Immunization Practices, JAMA 284 (6) (2000) 687-688.

[9] A.H. Reid, T.G. Fanning, T.A. Janczewski, J.K. Taubenberger, Characterization of the 1918 "Spanish" influenza virus neuraminidase gene, Proc. Natl. Acad. Sci. U. S. A. 97 (12) (2000) 6785-6790.

[10] A.D. Langmuir, The surveillance of communicable diseases of national importance, N. Engl. J. Med. 268 (1963) $182-192$.

[11] R.M. Zweighaft, D.W. Fraser, M.A.W. Hattwick, W.G. Winkler, W.C. Jordan, M. Alter, M. Wolfe, H. Wulff, K.M. Johnson, Lassa fever: response to an imported case, N. Engl. J. Med. 297 (1977) 803-807.

[12] S.B. Thacker, R.L. Berkelman, Public health surveillance in the United States, Epidemiol. Rev. 10 (1988) $164-190$

[13] M.S. Gottlieb, R. Schroff, H.M. Schanker, et al., Pneumocystis carinii pneumonia and mucosal candidiasis in previous healthy homosexual men: evidence of a new acquired cellular immunodeficiency, N. Engl. J. Med. 305 (1981) 1425.

[14] C.C. King, Infectious disease surveillance, in: C.H. Chiou (Ed.), Public Health Textbook, HwaHsin Publisher, Taipei, Taiwan, 1999, pp. 222-250 (in Chinese).

[15] G. Pugliese, M.S. Favero, Surveillance of unexplained illness and death, Inf. Control Hosp. Epidemiol. 19 (1998) 285-286.

[16] A.W. Hampson, N.J. Cox, Global surveillance for pandemic influenza: are we prepared?, in: L.E. Brown, A.W. Hampson, R.G. Webster (Eds.), Options for the Control of Influenza III, Elsevier, Amsterdam, 1996.

[17] E.C.J. Classs, OsterhausR.V. Beek, J.C. Jong, G.F. Rimmelzwaan, D.A. Senne, S. Krauss, K.F. Shortridge, R.G. Webster, Human influenza A H5N1 virus related to a highly pathogenic avian influenza virus, Lancet 351 (1998) 472-477.

[18] Y.J. Guo, S. Kraus, D.A. Senne, I.P. Mo, K.S. Lo, X.P. Xiong, M. Norwood, K.F. Shortridge, R.G. Webster, Characterization of the pathogenicity of members of the newly established H9N2 influenza virus lineage in Asia, Virology 267 (2000) 279-288. 
[19] Y. Guan, K.F. Shortridge, S. Krauss, R.G. Webster, Molecular characterization of H9N2 influenza viruses: were they the donors of the "internal" genes of H5N1 viruses in Hong Kong?, Proc. Natl. Acad. Sci. U. S. A. 96 (1999) 9363-9367.

[20] R.K. Tseng, H.Y. Chen, C.B. Horng, Influenza virus infections in Taiwan from 1979-1994, J. Formosan Med. Assoc. 94 (1995) S126-S136.

[21] H.K. Shieh, W.J. Huang, J.H. Shien, S.Y. Chiu, L.F. Lee, Y.S. Lu, Studies on avian influenza in Taiwan, R.O.C. (III): isolation, identification and pathogenicity tests of the virus isolated from breeding chickens, Taiwan J. Vet. Med. Anim. Husb. 59 (1992) 45-55.

[22] C.P. Chang, A.E. New, G.S. Irving, H.S. Chiang, J.F. Taylor, A surveillance of human influenza virus in swine in southern Taiwan, Int. J. Zoonoses 4 (1977) 25-30.

[23] M.C. Cheng, Wild bird influenza A virologic surveillance in Taiwan, Sept. 1998-Dec. 1999, Options for the Control; of Influenza IV Conference, Abstract \#p2-84, Crete, Greece, p. 152.

[24] M.S. Lee, Typing and subtyping of avian influenza viruses by reverse transcriptase polymerase chain reaction (RT-PCR), Master Degree Thesis, 1999, National Chung-Hsing University, Taichung, Taiwan, R.O.C.

[25] H.L. Yen, Epidemiological study of interspecies transmission of avian influenza viruses in Taiwan, Master Degree Thesis 2000, Institute of Epidemiology, College of Public Health, National Taiwan University, Taipei, Taiwan, R.O.C.

[26] C.P. Tsai, M.C. Cheng, D.T. Lin, C.M. Chen, The prevalence of hemaglutination-inhibition (HI) antibodies to influenza viruses A/swine/Iowa/15/30 (H1N1) and A/Swine/Obihiro/10/85 (H3N2) in Taiwan Pigs, July 1993 - Mar. 2000, Proc. 16th IPVS Congress Melbourne, Australia, 257.

[27] J.P. Hsu, Sentinel Physician Surveillance System Plan, Government Report 1999, Center for Disease Control, Department of Health, Taipei, Taiwan.

[28] D.P. Liu, Influenza Laboratory Surveillance., Government Report 2000, Center for Disease Control, Department of Health, Taipei, Taiwan.

[29] G. Webster Robert, Antigenic variation in influenza viruses, in: E. Domingo, R. Webster, J. Holland (Eds.), Origin and Evolution of Viruses, Academic Press, San Diego, 1999, pp. 377-390 (Chap. 14).

[30] T. Ito, Interspecies transmission and receptor recognition of influenza A viruses, Microbiol. Immunol. 44 (6) (2000) 423-430.

[31] E. Hoffmann, J. Stech, I. Leneva, S. Krauss, C. Scholtissek, P.S. Chin, M. Peris, K.F. Shortridge, R.G. Webster, Characterization of the influenza A virus gene pool in avian species in southern China: was H6N1 a derivative or a precursor of H5N1?, J. Virol. 74 (14) (2000) 6309-6315.

[32] D.P. Offringa, V. Tyson-Medlock, Z. Ye, R.A. Levandowski, A comprehensive systematic approach to identification of influenza A virus genotype using RT-PCR and RFLP, J. Virol. Methods 88 (1) (2000) $15-24$.

[33] A.I. Karasin, M.M. Schutten, L.A. Cooper, C.B. Smith, K. Subbarao, G.A. Anderson, S. Carman, C.W. Olsen, Protein, Nucleotide Genetic characterization of H3N2 influenza viruses isolated from pigs in North America, 1977-1999: evidence for wholly human and reassortant virus genotypes, Virus Res. 68 (1) (2000) 71-85 (Jun). 